\title{
Flow equation analysis of the anisotropic Kondo model
}

\author{
W. Hofstetter ${ }^{(1)}$ and S. Kehrein ${ }^{(2)}$ t \\ (1) Theoretische Physik III, Elektronische Korrelationen und Magnetismus, Universität Augsburg, 86135 Augsburg, Germany \\ ${ }^{(2)}$ Lyman Laboratory of Physics, Harvard University, Cambridge, MA 02138
}

(November 9, 2018)

\begin{abstract}
We use the new method of infinitesimal unitary transformations to calculate zero temperature correlation functions in the strong-coupling phase of the anisotropic Kondo model. We find the dynamics on all energy scales including the crossover behaviour from weak to strong coupling. The integrable structure of the Hamiltonian is not used in our approach. Our method should also be useful in other strong-coupling models since few other analytical methods allow the evaluation of their correlation functions on all energy scales.
\end{abstract}

PACS: 75.20.Hr, 11.10.Gh, 11.10.Hi

\section{INTRODUCTION}

The Kondo model, originally introduced by Zener [1], is the most basic Hamiltonian describing the physics of dilute magnetic impurities in metals. Despite its apparent simplicity, it has become a paradigm for complex many-body effects, due to its non-trivial strong-coupling behaviour at low temperatures. Unprecedented theoretical activity during the last four decades was triggered by the seminal work of Kondo [2], who showed that perturbation theory for an antiferromagnetic exchange coupling diverges at low temperatures. Within perturbative scaling [3 5] the coupling constant grows continuously and eventually diverges. This indicates that the impurity spin is screened in the ground state. However, the diverging coupling constant also implies the breakdown of the perturbative scaling approach in the strong-coupling phase. Therefore perturbative scaling does not provide a systematic and controlled expansion describing the weak to strong-coupling crossover. This is a typical situation in many strong-coupling problems. The development of new, nonperturbative methods like Wilson's numerical renormalization group (NRG) [6] and the Bethe ansatz [7] eventually led to a quantitative understanding of the strong-coupling regime in the Kondo model. Despite these successes, however, a simple analytical description of the crossover from a free spin to the Fermi liquid regime in an RG type framework was still missing.

In the present work, we will attempt to fill this gap by applying Wegner's flow equation method [8], which was recently employed successfully to diagonalize a related strong-coupling problem, the quantum sineGordon model [9]. We will mainly focus on the calculation of equilibrium dynamical correlation functions at zero temperature as this provides one of the most interesting new perspectives for this new approach. The calculation of correlation functions throughout the crossover region is notoriously difficult with exact methods building on the integrability of the model. There is considerable interest in theoretical tools that allow their deter- mination in strong-coupling models, which provides the main motivation for our work 10]. Also the integrability of the Kondo model is not used in our method and nonintegrable perturbations can be studied as well.

We consider the anisotropic Kondo Hamiltonian

$$
\begin{aligned}
H= & \sum_{k} \epsilon_{k} c_{k \alpha}^{\dagger} c_{k \alpha}+J_{\|} c_{0 \alpha}^{\dagger} \sigma_{\alpha \beta}^{z} c_{0 \beta} S^{z} \\
& +\frac{J_{\perp}}{2}\left(c_{0 \alpha}^{\dagger} \sigma_{\alpha \beta}^{+} c_{0 \beta} S^{-}+\text {h.c. }\right) .
\end{aligned}
$$

Here $\vec{S}$ is a spin- $1 / 2$ degree of freedom and $c_{0 \alpha}^{(\dagger)}=$ $\sum_{k} c_{k}^{(\dagger)} / \sqrt{L}$ are creation and annihilation operators for the localized electron state at the spin site. Notice that the anisotropic Kondo Hamiltonian is equivalent to the dissipative two-state system (spin boson model) [11, 12. For the noninteracting conduction band we assume a linear dispersion. We will treat the model in its bosonized form 13]: One introduces the bosonic spin density modes $\sigma(p)=\frac{1}{\sqrt{2|p|}} \sum_{q}\left(c_{p+q \uparrow}^{\dagger} c_{q \uparrow}-c_{p+q \downarrow}^{\dagger} c_{q \downarrow}\right)$ with the commutator $\left[\sigma(-q), \sigma\left(q^{\prime}\right)\right]=\delta_{q q^{\prime}} L / 2 \pi$ for $q, q^{\prime}>0$. Here $L$ is the system size and all other nonvanishing commutators can be derived using $\sigma^{\dagger}(q)=\sigma(-q)$. Notice $\sigma(-q) \mid \Omega>=0$ on the ground state for $q>0$. The charge density modes in (11) decouple completely and one only has to look at the spin density part of (11) [14]

$H=H_{0}-\frac{J_{\|}}{\sqrt{2} 2 \pi} \partial_{x} \Phi(0) S^{z}+\frac{J_{\perp}}{4 \pi a}\left(e^{i \sqrt{2} \Phi(0)} S^{-}+\right.$h.c. $)$.

Here $H_{0}=\frac{2 \pi}{L} v_{F} \sum_{q>0} q \sigma(q) \sigma(-q)$. In the following we set $v_{F}=1$. The bosonic field is defined as [15]

$$
\Phi(x)=-\frac{2 \pi i}{L} \sum_{q \neq 0} \frac{\sqrt{|q|}}{q} e^{-i q x-a|q| / 2} \sigma(q) .
$$

The parameter $a>0$ generates the UV-regularization of our model. We are interested in its universal properties at energies $|E| \ll a^{-1}$. The longitudinal spin coupling can be eliminated by a unitary transformation $U=\exp \left(i \mu S^{z} \Phi(0)\right)$ with $\mu=J_{\|} / \sqrt{2} 2 \pi$. We arrive at 


$$
H=H_{0}+g_{0}\left(V\left(\lambda_{0}, 0\right) S^{-}+V\left(-\lambda_{0}, 0\right) S^{+}\right)
$$

with the coupling constant $g_{0}=J_{\perp} / 4 \pi a$ and $\lambda_{0}=\sqrt{2}-$ $J_{\|} / \sqrt{2} 2 \pi$. Here vertex operators have been introduced

$$
V(\lambda, x) \equiv \exp (i \lambda \Phi(x))
$$

which are complicated many-body objects for a general value of the scaling dimension $\lambda$ 15. Notice that our $V(\lambda, x)$ are not normal-ordered. We will also need the Fourier-transformed normalized vertex operators $C_{p}^{\dagger}=\alpha_{p}^{-1} L^{-1 / 2} \int d x e^{i p x} V(\lambda, x)$ with $\alpha_{p}^{2}=$ $2 \pi a|p a|^{\lambda^{2}-1} / \Gamma\left(\lambda^{2}\right)$. Notice $C_{-p}^{\dagger}|\Omega\rangle=C_{p}|\Omega\rangle=0$ for $p>0$, and $\left\langle\Omega\left|C_{p} C_{p^{\prime}}^{\dagger}\right| \Omega\right\rangle=\delta_{p p^{\prime}} \theta(p),\left\langle\Omega\left|C_{p}^{\dagger} C_{p^{\prime}}\right| \Omega\right\rangle=$ $\delta_{p p^{\prime}} \theta(-p)$ for $|a p| \ll 1$.

Eq. (2) with general initial couplings $\lambda_{0}$ and $g_{0}$ is our starting point for the flow equation approach. The universal low-energy properties of (2) are equivalent to those of the spin-density part of (1D). As proposed by Wegner [8], we apply a sequence of infinitesimal unitary transformations to (2) in order to make it successively more diagonal. One can set this up in a differential formulation

$$
\partial_{B} H(B)=[\eta(B), H(B)]
$$

with an anti-Hermitian generator $\eta(B) . H(B=0)$ is our initial Hamiltonian (2) and all $H(B)$ as obtained by the solution of (3) are unitarily equivalent to it. In general we will have to truncate our system of equations, so this equivalence will only be approximate. In order to generate a stable sequence of approximations, we choose $\eta(B)$ such that first (for small $B$ ) offdiagonal matrix elements are removed which couple states with large energy differences (of order $B^{-1 / 2}$ ), and later more degenerate states. This is reminiscent of the energy scale separation underlying perturbative scaling. Similar ideas have independently been developed by Głazek and Wilson (similarity renormalization scheme) [16] that contain Wegner's method as a special case.

As the flow parameter $B$ grows, the effective spin flip interaction in the interaction part $H_{\text {int }}(B)$ will become increasingly nonlocal and the scaling dimension of the vertex operator will begin to flow. We take this into account by writing

$$
H(B)=H_{0}+\int d x g(B, x)\left(V(\lambda(B), x) S^{-}+\text {h.c. }\right)
$$

with $g(B, x)=L^{-1 / 2} \sum_{p} g_{p}(B) e^{i p x}$. Wegner has suggested the generic choice $\eta^{(1)}=\left[H_{0}, H_{\text {int }}\right]$ for the generator, as this always removes the off-diagonal matrix elements in the above energy-scale separated way [8]. Our generator has the following more general form

$$
\begin{aligned}
\eta= & \sum_{p} \eta_{p}^{(1)}\left(C_{p}^{\dagger} S^{-}-C_{p} S^{+}\right) \\
& +\sum_{p, q} \eta_{p q}^{(2)}\left(C_{p}^{\dagger} C_{q}-C_{q} C_{p}^{\dagger}\right)
\end{aligned}
$$

Here $\eta_{p}^{(1)}=p \alpha_{p} g_{p}$ follows from Wegner's canonical choice. The second generator part has been introduced since it will later allow us to generate transformed Hamiltonians with a particularly simple structure. The precise form of $\eta_{p q}^{(2)}$ will be discussed below.

In general, new many-particle interactions are generated in (3). Our truncation criterion is like in [9] the operator product expansion (OPE) for vertex operators. For example in the anticommutator of vertex operators the OPE leads to 15

$$
\begin{aligned}
& \{V(\lambda, x), V(-\lambda, y)\}=\left(1+i \lambda(x-y) \partial_{x} \Phi(x)+\ldots\right) \\
& \times\left(\frac{1}{[1+i(x-y) / a]^{\lambda^{2}}}+\frac{1}{[1-i(x-y) / a]^{\lambda^{2}}}\right) .
\end{aligned}
$$

We only keep the leading nonvanishing term of this expansion in our calculation. This amounts to neglecting terms with larger scaling dimensions (more irrelevant terms) and is therefore reminiscent of an RG approach. Higher order terms can be successively taken into account in a systematic expansion. However, the present approximation will already turn out to lead to very accurate results. Within our approximation, the primary effect of the flow (3) is to generate a new interaction term

$$
H_{\text {new }}^{(1)}=S^{z} \int d x f(x) \partial_{x} \Phi(x)
$$

in (何) with a function $f(x)$ that depends on the parameters $g_{p}$. (6) is identical to the initial longitudinal spin coupling. Our strategy is to eliminate $H_{\text {new }}^{(1)}$ after it is generated infinitesimally by again performing a unitary transformation $U=\exp \left[i \epsilon S^{z} \Phi(0)\right]$ with a suitable infinitesimal $\epsilon$. Similar to the initial value of the scaling dimension that is determined by the longitudinal Kondo coupling, we find a further shift in $\lambda(B)$ due to the elimination of $H_{\text {new }}^{(1)}$ : one derives the following flow equation

$$
\partial_{B} \lambda^{2}=\frac{8 \pi a \lambda^{2}\left(1-\lambda^{2}\right)}{\Gamma\left(\lambda^{2}\right)} \sum_{p} g_{p} g_{-p}|p a|^{\lambda^{2}-1} .
$$

In the sequel we will only look at the strong-coupling phase $\left(\left|J_{\perp}\right|>-J_{\|}\right.$for small couplings $\left.\left|J_{\perp}\right| \ll 1\right)$ since the weak coupling regime is trivial. In the strong-coupling phase the flow is always directed towards the Toulouse line $\lambda=1$ [17], which constitutes the strong-coupling fixed point of our approach (see fig. 1). For $\lambda=1$ the vertex operators in (2) obey fermionic anticommutation relations [15] and the Hamiltonian is quadratic (equivalent to a noninteracting resonant level model [17]): The OPE series (5) terminates after the leading term and the flow equations close exactly since they can readily diagonalize a quadratic Hamiltonian. We flow to a point where our method becomes exact, thereby avoiding the usual strong-coupling divergence. This is the key improvement of our approach as compared to perturbative scaling, which does not make use of the special properties at $\lambda=1$. 


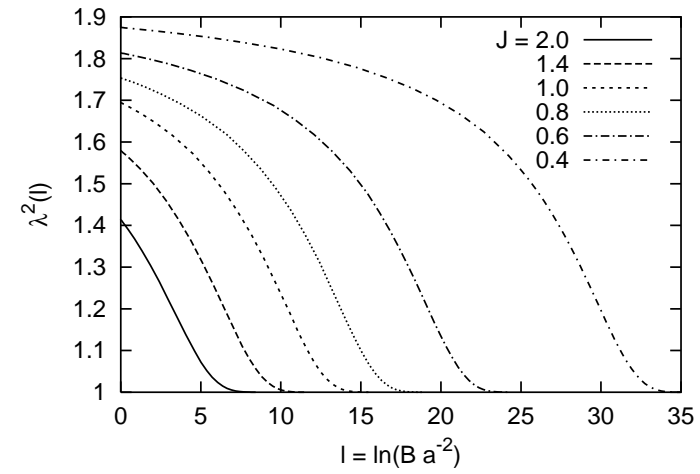

FIG. 1. Flow of the scaling dimension for various couplings $J$ in the isotropic Kondo model.

The second new interaction that is generated is a potential scattering term. Only diagonal terms appear

$$
H_{\text {new }}^{(2)}(B)=\sum_{p} \omega_{p}(B)\left(C_{p}^{\dagger} C_{p}-C_{p} C_{p}^{\dagger}\right)
$$

with the following choice of the generator $\eta_{p q}^{(2)}(B)$

$$
\eta_{p q}^{(2)}=\frac{1}{2} \frac{(p+q) \alpha_{p} \alpha_{q} g_{p} g_{q}}{p-q+2\left(\omega_{p}-\omega_{q}\right)} .
$$

$H_{\text {new }}^{(2)}(B=\infty)$ is a term in order $1 / L$ since $\omega_{p}(B)=$ $O(1 / L)$ and thus represents the only "impurity effect" in the Hamiltonian after elimination of the Kondo coupling for $B \rightarrow \infty$ in our present approximation

$$
H(B=\infty)=H_{0}+H_{\text {new }}^{(2)}(B=\infty) .
$$

Together with (7) we thus obtain a closed set of flow equations

$$
\begin{aligned}
\partial_{B} g_{p}= & -p^{2} g_{p}+2 \sum_{q} \frac{\alpha_{q}}{\alpha_{p}} \eta_{p q}^{(2)} g_{q} \\
& +\frac{1}{2} g_{p} \ln \left(\frac{B}{a^{2}}\right) \lambda \partial_{B} \lambda-2 p g_{p} \omega_{p} \\
\partial_{B} \omega_{p}= & p \alpha_{p}^{2} g_{p}^{2} .
\end{aligned}
$$

Neglecting the momentum dependence induced by $\eta^{(2)}$ and the potential scattering terms $\omega_{p}$ (which become important only at energies of the order of the Kondo scale), we can parametrize the solution of (8) as $g_{p}(B)=$ $\tilde{g}(B) e^{-p^{2} B}$ with the running coupling $\tilde{g}(B) . \tilde{g}(B)$ is in general strongly renormalized. In the low energy limit the scaling dimension approaches $\lambda=1$ and we can then deduce the Kondo scale from the effective Toulouse Hamiltonian where $T_{K} \sim \tilde{g}(B=\infty)^{2} / a$. For the symmetric case $J_{\|}=J_{\perp}=J$ and a small antiferromagnetic coupling $0<J \ll 1$ one e.g. shows $T_{K} \sim \frac{J^{\tau}}{a} \exp \left(-\frac{2 \pi}{J}\right)$, where $\tau=(1+\gamma-\ln 2) / 3 \approx 0.295$. NRG and third order scaling results by Wilson $[6]$ predict the same leading exponential behaviour but a slightly different prefactor with $\tau=1 / 2[18]$.
Our main focus in this work is the calculation of the dynamical impurity susceptibility $\chi(t)=$ $i \theta(t)\left\langle\left[S^{z}(t), S^{z}(0)\right]\right\rangle$ at zero temperature. The key ideas for the calculation of correlation functions for impurity systems within the flow equation approach have been developed in [19]: we need to evaluate matrix elements of the observable $O=S^{z}$ with respect to the eigenstates of $H(B=\infty)$, which is simplified by the fact that the final Hamiltonian is diagonal. In order to make use of this simplification, we need to perform the same series of infinitesimal unitary transformations on $O$ as on $H(B)$

$$
\partial_{B} O(B)=[\eta(B), O(B)] .
$$

Usually, keeping the Hamiltonian flow (3) simple leads to a complicated structure of the transformed observable. In the present case, however, it turns out that the OPE can be used as a unifying truncation criterion for both $H(B)$ and $O(B)$. Using $S^{z}=\left[S^{+}, S^{-}\right] / 2$ and the ansatz

$$
S^{+}(B)=h(B) S^{+}+S^{z} \sum_{p} d_{p}(B) C_{p}^{\dagger}
$$

for the flow of the observable [20] another closed set of equations is obtained:

$$
\begin{aligned}
\partial_{B} h & =\frac{1}{2} \sum_{p} p \alpha_{p} d_{p} g_{p} \\
\partial_{B} d_{p} & =-2 p g_{p} \alpha_{p} h+2 \sum_{q} \eta_{p q}^{(2)} d_{q} .
\end{aligned}
$$

We have numerically solved this system of equations in combination with (8). It is known from the theory of dissipative quantum systems 19 that in the thermodynamic limit the impurity observable $S^{+}$decays completely, i.e. $h(B=\infty)=0$. With $S^{z}(B=\infty)$ completely expressed in terms of band operators, it is then trivial to calculate the dynamical susceptibility: since $H_{\text {new }}^{(2)}(B=\infty)$ is of order $1 / L$, it does not contribute to the dynamics and we need only study the time evolution of $S^{z}(B=\infty)$ under $H_{0}$. The imaginary part of the Fourier-transform of $\chi(t)$ follows easily

$$
\begin{aligned}
\chi^{\prime \prime}(\omega)=\frac{\pi}{16} \sum_{p, q>0} d_{p}^{2}(B=\infty) d_{q}^{2}(B=\infty) \\
\quad \times\left(\delta\left(\omega-\epsilon_{p}-\epsilon_{q}\right)-\delta\left(\omega+\epsilon_{p}+\epsilon_{q}\right)\right) .
\end{aligned}
$$

Results are shown in fig. 2: The curves display a broad maximum at an energy of the order of the Kondo scale and a power law decay $\chi^{\prime \prime}(\omega) \sim \omega^{-(3-2 \alpha)}$ at high frequencies, consistent with results obtained for the spin boson model [21]. At low frequencies one finds $\chi^{\prime \prime}(\omega) \sim \omega$, where the corresponding slope scales as $T_{K}^{-2}$ with a prefactor of order one 22. The normalization condition $\int_{0}^{\infty} d \omega \chi^{\prime \prime}(\omega)=\pi / 4$ is fulfilled due to the sum rule $h^{2}(B)+\sum_{p} d_{p}^{2}(B) / 4=1$ that holds exactly [23]. 


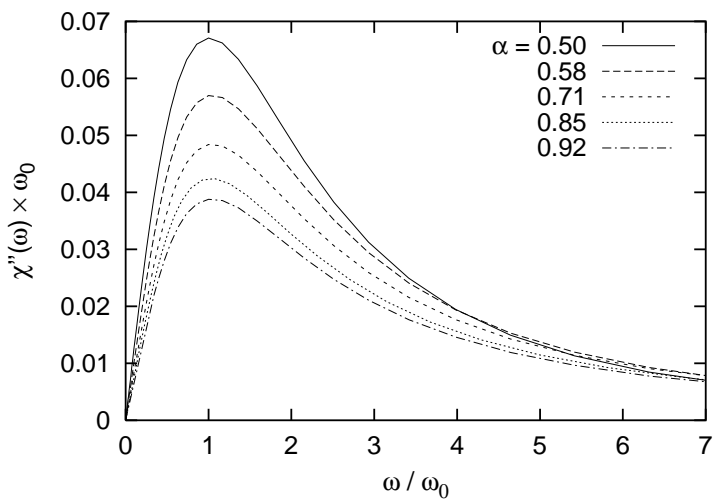

FIG. 2. Universal scaling forms of the dynamical impurity susceptibility for different dissipation strengths $\alpha=\left(1-J_{\|} / 4 \pi\right)^{2}$ in the limit of small coupling $J_{\perp}: \omega_{0}$ is defined by the maximum of the curves. One finds $\omega_{0} \propto T_{K}$.

The real part $\chi^{\prime}(\omega)$ can be obtained by a KramersKronig transformation. In particular, the static susceptibility in response to a local field is given by $\chi_{0}=\chi^{\prime}(\omega=$ 0 ) and therefore contains spectral information on all energy scales. In the limit of small spin flip coupling $J_{\perp}$ we have numerically verified the power law behaviour $\chi_{0} \sim J_{\perp}^{1 /(\alpha-1)}$ known from the ohmic spin boson model with the dissipation parameter $\alpha=\left(1-J_{\|} / 4 \pi\right)^{2}$ [21].

Summing up, we have applied Wegner's flow equations to the anisotropic Kondo problem. The Hamiltonian is written in terms of vertex operators with scaling dimensions that flow to the Toulouse point. In contrast to the perturbative scaling approach, our flow equations close exactly at the Toulouse point with finite couplings and a nontrivial strong-coupling behaviour. Similar to the analysis of the sine-Gordon model [9] no strongcoupling divergence of the coupling constants is encountered. We are thus able to give an analytic description of the crossover from weak to strong coupling in a systematic expansion that can be improved by taking higher orders in the OPE into account. The stability of the strong-coupling fixed point cannot be endangered in this expansion.

In our solution of the flow equations we have focussed on the equilibrium dynamics of the impurity at zero temperature. As an example we have calculated the universal scaling forms of the local dynamical susceptibility $\chi(\omega)$ and found good agreement with results known from the spin-boson model. This exemplifies the usefulness of our approach for the calculation of correlation functions in strong-coupling problems, and should be of interest in other models as well. Notice that we have not used the integrable structure of the Kondo model in our method. Future work will concentrate on the effect of finite temperature, and on the calculation of static impurity contributions where the newly generated potential scattering terms give the leading contribution (in contrast to the dynamical susceptibility where these terms play no role) and our present approximation must be improved.

The authors acknowledge many valuable discussions with T.A. Costi, D. Fisher, A. Mielke and D. Vollhardt. This work was supported by the Deutsche Forschungsgemeinschaft (DFG), by the SFB 484 of the DFG and by the National Science Foundation (NSF) under grants DMR 9630064, DMR 9976621 and DMR 9981283.

* Present address: Theoretische Physik III, Universität Augsburg, 86135 Augsburg, Germany.

[1] C. Zener, Phys. Rev. 81, 440 (1951).

[2] J. Kondo, Prog. Theor. Phys. 32, 37 (1964).

[3] P.W. Anderson and G. Yuval, Phys. Rev. Lett. 23, 89 (1969).

[4] P.W. Anderson, G. Yuval, and D.R. Hamann, Phys. Rev. B1, 4464 (1970).

[5] P.W. Anderson, J. Phys. C3, 2436 (1970).

[6] K.G. Wilson, Rev. Mod. Phys. 47, 773 (1975).

[7] A.M. Tsvelick and P.B. Wiegmann, Adv. Phys. 32, 453 (1983).

[8] F. Wegner, Ann. Physik 3, 77 (1994).

[9] S. Kehrein, Phys. Rev. Lett. 83, 4914 (1999); Preprint cond-mat/0006403, to appear in Nucl. Phys. B.

[10] For an alternative analytical approach see F. Lesage, H. Saleur, and S. Skorik, Phys. Rev. Lett. 76, 3388 (1996); Nucl. Phys. B474, 602 (1996).

[11] F. Guinea, V. Hakim, and A. Muramatsu, Phys. Rev. B32, 4410 (1985).

[12] A.J. Leggett, S. Chakravarty, A.T. Dorsey, M.P.A. Fisher, A. Garg and W. Zwerger, Rev. Mod. Phys. 59, 1 (1987).

[13] K.D. Schotte, Z. Phys. 230, 99 (1970).

[14] The Klein factors can be absorbed into redefined spin operators and are of no concern in the sequel.

[15] For an overview of properties of vertex operators see e.g. J. v. Delft and H. Schöller, Ann. Physik 7, 225 (1998).

[16] S. D. Głazek and K. G. Wilson, Phys. Rev. D48, 5863 (1993) and Phys. Rev. D49, 4214 (1994).

[17] G. Toulouse, C. R. Acad. Sci. Paris 268, 1200 (1969).

[18] Possible reasons for this discrepancy are either the neglected higher order OPE terms, or the different cutoff schemes in the fermionic and bosonized models.

[19] S. Kehrein and A. Mielke, Ann. Physik (Leipzig) 6, 90 (1997); J. Stat. Phys. 90 (1998) 889.

[20] Again more irrelevant terms are neglected in this ansatz, which becomes exact on the Toulouse line.

[21] T.A. Costi and G. Zarand, Phys. Rev. B59, 12398 (1999).

[22] This prefactor of order one is not obtained correctly in the present approach. However, this discrepancy occurs on an energy scale $\ll \omega_{0}$ and cannot be resolved in fig. 2 . The deviation is due to our approximations in this work and will be improved in a subsequent publication.

[23] F. Wegner, private communication. 